\title{
Fast Normal Basis Multiplication Using General Purpose Processors (Extended Abstract)
}

\author{
Arash Reyhani-Masoleh ${ }^{1}$ and M. Anwar Hasan ${ }^{2}$ \\ 1 Centre for Applied Cryptographic Research, \\ Department of Combinatorics and Optimization, \\ University of Waterloo, Waterloo, Ontario, Canada N2L 3G1. \\ areyhani@cacr.math. uwaterloo.ca \\ 2 Department of Electrical and Computer Engineering, \\ University of Waterloo, Waterloo, Ontario, Canada N2L 3G1. \\ ahasan@ece.uwaterloo.ca
}

\begin{abstract}
For cryptographic applications, normal bases have received considerable attention, especially for hardware implementation. In this article, we consider fast software algorithms for normal basis multiplication over the extended binary field $\operatorname{GF}\left(2^{m}\right)$. We present a vector-level algorithm which essentially eliminates the bit-wise inner products needed in the conventional approach to the normal basis multiplication. We then present another algorithm which significantly reduces the dynamic instruction counts. Both algorithms utilize the full width of the data-path of the general purpose processor on which the software is to be executed. We also consider composite fields and present an algorithm which can provide further speed-up and an added flexibility toward hardwaresoftware co-design of processors for very large finite fields.
\end{abstract}

Keywords: Finite field multiplication, normal basis, software algorithms, ECDSA, composite fields.

\section{Introduction}

The extended binary finite field $\mathrm{GF}\left(2^{m}\right)$ of degree $m$ is used in important cryptographic operations, such as, key exchange, signing and verification. For today's security applications the minimum values of $m$ are considered to be 160 in the elliptic curve cryptography and 1024 in the standard discrete log based cryptography. Elliptic curve crypto-systems use relatively smaller field sizes, but require considerable amount of field arithmetic for each group operation (i.e., addition of two points). In such crypto-systems, often the most complicated and expensive module is the finite field arithmetic unit. As a result, it is important to develop suitable finite field arithmetic algorithms and architectures that can meet the constraints of various implementation technologies, such as, hardware and software.

S. Vaudenay and A. Youssef (Eds.): SAC 2001, LNCS 2259, pp. 230244 2001.

(C) Springer-Verlag Berlin Heidelberg 2001 
For cryptographic applications, the most frequently used $\mathrm{GF}\left(2^{m}\right)$ arithmetic operations are addition and multiplication. Compared to the former, the latter is much more complicated and time consuming operation. The complexity of $\mathrm{GF}\left(2^{m}\right)$ multiplication depends very much on how the field elements are represented. For hardware implementation of a multiplier, the use of normal bases has received considerable attention and a number of hardware architectures and implementations have been reported (see for example [1, [2, [7, 20]). A majority of such efforts were motivated by the fact that certain normal bases, e.g., optimal bases, yield area efficient multipliers, and that the field squaring, which is heavily used in exponentiation and Frobenius mapping, is a simple cycle shift of the field element's coordinates and hence in hardware it is almost free of cost. However, the task of implementing a normal basis multiplier in hardware poses a number of challenges. For example, when one has to deal with very large fields, the interconnections among the various parts of the multiplier could be quite irregular which may slow down the clock speed. Also, normal basis multipliers are not easily scalable with $\mathrm{m}$. Given a normal basis multiplier designed for $\mathrm{GF}\left(2^{233}\right)$, one cannot conveniently make it usable for $\mathrm{GF}\left(2^{163}\right)$ or $\mathrm{GF}\left(2^{283}\right)$.

Unlike hardware, so far software implementation of a $\mathrm{GF}\left(2^{m}\right)$ multiplier using normal bases has not been very efficient. This is mainly due to a number of practical considerations. Most importantly, normal basis multiplication algorithms require inner products or matrix multiplications over the ground field GF(2). Such computations are not directly supported by most of today's general purpose processors. These computations require bit-by-bit logical AND and XOR operations, which are not efficiently implemented using the instruction set supported by the processors. Also, when a high level programming language, such as, $\mathrm{C}$ is used, the cyclic shifts needed for field squaring operations, are not as efficient as they are in hardware.

In this article, we consider algorithms for fast software normal basis multiplication on general purpose processors. We discuss how the conventional bit-level algorithm for normal basis multiplication fails to utilize the full data-path of the processor and makes its software implementation inefficient. We then present a vector-level normal basis multiplication algorithm which eliminates the matrix multiplication over GF(2) and significantly reduces the number of dynamic instructions. We then derive another scheme for normal basis multiplication to further improve the speed. We also consider normal basis multiplication over certain special classes of composite fields. We show that normal basis multipliers over such composite fields can provide an additional speed-up and a great deal of flexibility toward hardware-software co-design of very large finite field processors.

\section{Preliminaries}

\subsection{Normal Basis Representation}

It is well known that there exists a normal basis (NB) in the field $G F\left(2^{m}\right)$ over $G F(2)$ for all positive integers $m$. By finding an element $\beta \in G F\left(2^{m}\right)$ such that 
$\left\{\beta, \beta^{2}, \cdots, \beta^{2^{m-1}}\right\}$ is a basis of $G F\left(2^{m}\right)$ over $G F(2)$, any element $A \in G F\left(2^{m}\right)$ can be represented as $A=\sum_{i=0}^{m-1} a_{i} \beta^{2^{i}}=a_{0} \beta+a_{1} \beta^{2}+\cdots+a_{m-1} \beta^{2^{m-1}}$, where $a_{i} \in G F(2), 0 \leq i \leq m-1$, is the $i$-th coordinate of $A$. In this article, this normal basis representation of $A$ will be written in short as $A=\left(a_{0}, a_{1}, \cdots, a_{m-1}\right)$. In vector notation, element $A$ will be written as $A=\underline{a} \cdot \underline{\beta}^{T}=\underline{\beta} \cdot \underline{a}^{T}$, where $\underline{a}=$ $\left[a_{0}, a_{1}, \cdots, a_{m-1}\right], \underline{\beta}=\left[\beta, \beta^{2}, \cdots, \beta^{2^{m-1}}\right]$, and $T$ denotes vector transposition. Now, consider the following matrix

$$
\mathbf{M}=\underline{\beta}^{T} \cdot \underline{\beta}=\left[\beta^{2^{i}+2^{j}}\right]_{i, j=0}^{m-1},
$$

whose entries belong to $\mathrm{GF}\left(2^{m}\right)$. Writing these entries with respect to the NB, one obtains the following.

$$
\mathbf{M}=\mathbf{M}_{0} \beta+\mathbf{M}_{1} \beta^{2}+\cdots+\mathbf{M}_{m-1} \beta^{2^{m-1}}
$$

where $\mathbf{M}_{i}$ 's are $m \times m$ multiplication matrices whose entries belong to $G F(2)$. Let $H\left(\mathbf{M}_{i}\right), 0 \leq i \leq m-1$, be the number of 1's (or Hamming weight) of $\mathbf{M}_{i}$. It is easy to verify that $H\left(\mathbf{M}_{0}\right)=H\left(\mathbf{M}_{1}\right)=\cdots=H\left(\mathbf{M}_{m-1}\right)$. The number of logic gates needed for the implementation of a NB multiplier depends on $H\left(\mathbf{M}_{i}\right)$ which is referred to as the complexity of the normal basis. Let us denote this complexity as $C_{N}$. It was shown in 12 that $C_{N} \geq 2 m-1$. When $C_{N}=2 m-1$, the NB is called an optimal normal basis (ONB).

Two types of ONBs were constructed by Mullin et al. [12. Gao and Lenstra [5] showed that these two types are all the ONBs in $G F\left(2^{m}\right)$. As an extension of the work on ONBs, Ash et al. in 3] proposed low complexity normal bases of type $t$ where $t$ is a positive integer. These low complexity bases are referred to as Gaussian Normal Basis (GNB). When $t=1$ and 2, the GNBs become the two types of ONBs of [3]. A type $t$ GNB for $G F\left(2^{m}\right)$ exists if and only if $p=t m+1$ is prime and $\operatorname{gcd}\left(\frac{t m}{k}, m\right)=1$, where $k$ is the multiplicative order of 2 modulo $p$ [8]. More on this can be found in [3].

\subsection{Conventional NB Multiplication Algorithm}

Below we give the conventional normal basis multiplication algorithm as described by NIST in 13. This algorithm is for $t$ even only (the reader is referred to [8] for algorithm with $t$ odd). The case of $t$ even is of particular interest for implementing high speed crypto-systems based on Koblitz curves. Such curves with points over $G F\left(2^{m}\right)$ exist for $m=163,233,283,409,571$, where normal bases have $t$ even. Note that in the following algorithm, $p=t m+1$, and $A \ll i$ (resp. $A \gg i$ ) denotes $i$-fold left (resp. right) cyclic shifts of the coordinates of $A$. The algorithm requires the input sequence $F(1), F(2), \cdots, F(p-1)$ to be pre-computed using

$$
F\left(2^{i} u^{j} \bmod p\right)=i, 0 \leq i \leq m-1,0 \leq j<t,
$$

where $u$ is an integer of order $t \bmod p$. 
Algorithm 1 (Bit-Level NB Multiplication)

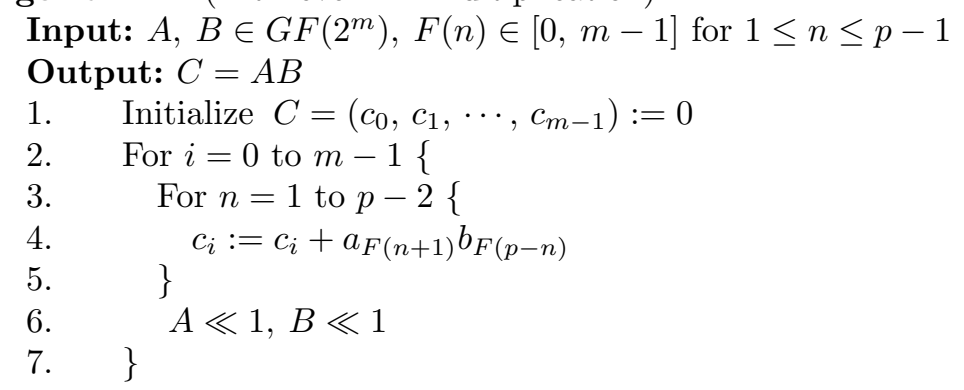

Software implementation of Algorithm 1 is not very efficient for the following reasons. First, in each execution of line 4 , one coordinate of each of $A$ and $B$ are accessed. These accesses are such that their software implementation is rather unsystematic and typically requires more than one instruction. Secondly, in line 4 the mod 2 multiplication of the coordinates, which is implemented by bit level logical AND operation, is performed $m(p-2)$ times in total, and the mod 2 addition, which is implemented by bit level logical XOR operation, is performed $\frac{1}{4} m(p-2)$ times, on average, assuming that $A$ and $B$ are two random inputs. In the $\mathrm{C}$ programming language, these mod 2 multiplication and addition operations correspond to about $m(p-2)$ AND and $\frac{1}{4} m(p-2)$ XOR instruction 1 , respectively.

\section{Vector-Level NB Multiplication}

In this section we discuss improvements to Algorithm 1 so that normal basis multiplication can be efficiently implemented in software. One crucial improvement is that most arithmetic operations are done on vectors instead of bits. This enables us to use the full data-path of the processor on which the software is executed. The assumption that $t$ is even in Algorithm 1 is also used in the remaining discussion of this section.

Lemma 1. For GNB of type $t$, where $t$ is even, the sequence $F(n)$ of $p-1$ integers as defined above is mirror symmetric around the center, i.e., $F(n)=$ $F(p-n), 1 \leq n \leq p-1$.

Proof. In (3), $t$ is the smallest nonzero integer such that $u^{t} \bmod p=1$. Then $u^{\frac{t}{2}} \bmod p$ must be equal to -1 . For $0 \leq i \leq m-1$ and $0 \leq j \leq t-1$, let $n=2^{i} u^{j} \bmod p$. Then $F(n)=F\left(2^{i} u^{j} \bmod p\right)=i$. Also, $F\left(2^{i} u^{\frac{t}{2}+j} \bmod p\right)=i$. Thus $F(n)=F\left(2^{i} u^{\frac{t}{2}+j} \bmod p\right)=F\left(-2^{i} u^{j} \bmod p\right)=F(p-n)$.

From (3i) and Lemma 1, one has $F(1)=F(p-1)=0$. For $1 \leq n \leq p-2$, let us define

$$
\Delta F(n)=F(n+1)-F(n) \bmod m .
$$

Now we have the following corollary.

${ }^{1}$ These are dynamic instructions which the underlying processor needs to execute. 
Corollary 1. For $\Delta F(n)$ as defined above and for $t$ even, the following holds

$$
\Delta F(p-n)=m-\Delta F(n-1) \bmod m, 1 \leq n \leq p-2 .
$$

Proof. Using (4), one obtains $F(n+1)=\sum_{i=1}^{n} \Delta F(i)$. Applying Lemma 1 into (4), one can also write $\Delta F(p-n)=-\Delta F(n-1) \bmod m, 2 \leq n \leq p-1$ which results in $\Delta F(p-n)=m-\Delta F(n-1), 2 \leq n<\frac{p-1}{2}$, and $\Delta F\left(\frac{p-1}{2}\right)=0$.

In Algorithm 1 the $i$-th coordinate of the product $C=A B$ is computed in its inner loop which can be written as follows

$$
c_{i}=\sum_{n=1}^{p-2} a_{F(n+1)+i} b_{F(p-n)+i}, 0 \leq i \leq m-1 .
$$

Using Lemma 1 and equation (4), one can write

$$
\begin{aligned}
c_{i} & =\sum_{n=1}^{p-2} a_{F(n+1)+i} b_{F(n)+i}, 0 \leq i \leq m-1, \\
& =\sum_{n=1}^{p-2} a_{F(n)+\Delta F(n)+i} b_{F(n)+i}, 0 \leq i \leq m-1 .
\end{aligned}
$$

For a particular GNB, the values of $\Delta F(n), 1 \leq n \leq p-2$, are fixed and are to be determined only once, i.e., at the time of choosing the basis. Additionally, Corollary 1 implies that it is sufficient to store only half (i.e., $\frac{p-1}{2}$ ) of these $\Delta F(n)$ 's. We now state the vector-level algorithm for $t$ even as follows. A similar algorithm for odd values of $t$ is given in [18].

Algorithm 2 (Vector-Level NB Multiplication)

$$
\begin{aligned}
& \text { Input: } A, B \in G F\left(2^{m}\right), \Delta F(n) \in[0, m-1], 1 \leq n \leq p-1 \\
& \text { Output: } C=A B \\
& \text { 1. } \quad \text { Initialize } S_{A}:=A, S_{B}:=B, C:=0 \\
& \text { 2. } \quad \text { For } n=1 \text { to } p-2\{ \\
& \text { 3. } \quad S_{A} \ll \Delta F(n) \\
& \text { 4. } \quad R:=S_{A} \odot S_{B} \\
& \text { 5. } \quad C:=C+R \\
& 6 . \quad \quad S_{B} \ll \Delta F(n) \\
& 7 . \quad\}
\end{aligned}
$$

In line 4 of Algorithm 2, for $X, Y \in G F\left(2^{m}\right), X \odot Y$ denotes the bit-wise AND operation between coordinates of $X$ and $Y$, i.e., $X \odot Y=\left(x_{0} y_{0}, x_{1} y_{1}, \cdots\right.$, $\left.x_{m-1} y_{m-1}\right)$. In order to obtain an overall computation time for a $G F\left(2^{m}\right)$ multiplication using Algorithm 2, the coordinates of the field elements can be divided into $\left\lceil\frac{m}{\omega}\right\rceil$ units where $\omega$ corresponds to the data-path width of the processor. We assume that the processor can perform bit-wise XOR and AND of two $\omega$-bit operands using one single XOR instruction and one single AND instruction, respectively. Since the loop in Algorithm 2, has $p-2$ iterations, the total number of 
bit-wise AND and bit-wise XOR instructions are the same and is $(p-2)\left\lceil\frac{m}{\omega}\right\rceil=$ $(t m-1)\left\lceil\frac{m}{\omega}\right\rceil$. Also, this algorithm needs $2(p-2)\left\lceil\frac{m}{\omega}\right\rceil=2(t m-1)\left\lceil\frac{m}{\omega}\right\rceil$ cyclic shifts. We assume that an $i$-fold, $1 \leq i<\omega$, left/right shift can be emulated in the $\mathrm{C}$ programming language using a total of $\rho$ instructions. The value of $\rho$ is typically 4 when simple logical instructions, such as AND, SHIFT, and OR are used. We can now state the following theorem.

Theorem 1. The dynamic instruction count for Algorithm 0 is given by

$$
\text { \#Instructions } \approx 2(1+\rho)(t m-1)\left\lceil\frac{m}{\omega}\right\rceil .
$$

\section{Efficient NB Multiplication over $G F\left(2^{m}\right)$}

In this section, we develop another algorithm for normal basis multiplication. We also analyze the cost of this algorithm in terms of dynamic instruction counts and memory requirements and then compare them with those of similar other algorithms.

\subsection{Algorithm}

For the normal basis $\left\{\beta, \beta^{2^{1}}, \cdots, \beta^{2^{m-1}}\right\}$, let $\delta_{j}=\beta^{1+2^{j}}, j=1, \cdots, v$, where $v=\left\lceil\frac{m-1}{2}\right\rceil$. Then one has the following result from [16].

Lemma 2. Let $A$ and $B$ be two elements of $G F\left(2^{m}\right)$ and $C$ be their product. Then

$$
C= \begin{cases}\sum_{i=0}^{m-1}\left[a_{i} b_{i} \beta^{2^{i+1}}+\left(\sum_{j=1}^{v} x_{i, j} \delta_{j}^{2^{i}}\right)\right], & \text { for m odd } \\ \sum_{i=0}^{m-1}\left[a_{i} b_{i} \beta^{2^{2+1}}+\left(\sum_{j=1}^{v-1} x_{i, j} \delta_{j}^{i}\right)+a_{i} b_{v+i} \delta_{v}^{2^{i}}\right], & \text { for m even }\end{cases}
$$

where $a_{i}$ 's and $b_{i}$ 's are the $N B$ coordinates of $A$ and $B$, respectively. Also, indices and exponents are reduced $\bmod m$ and

$$
x_{i, j}=a_{i} b_{i+j}+a_{i+j} b_{i}, \quad 1 \leq j \leq v, 0 \leq i \leq m-1 .
$$

Let $h_{j}, 1 \leq j \leq v$, be the number of 1's in the normal basis representation of $\delta_{j}$. Let $w_{j, 1}, w_{j, 2}, \cdots, w_{j, h_{j}}$ denote the positions of 1's in the normal basis representation of $\delta_{j}$, i.e.,

$$
\delta_{j}=\sum_{k=1}^{h_{j}} \beta^{2^{w_{j, k}}}, 1 \leq j \leq v,
$$

where $0 \leq w_{j, 1}<w_{j, 2}<\cdots<w_{j, h_{j}} \leq m-1$. Now, using (9) into Lemma 2, we have the following for $m$ odd.

$$
C=\sum_{i=0}^{m-1} a_{i} b_{i}{\beta^{2}}^{i+1}+\sum_{i=0}^{m-1} \sum_{j=1}^{v} x_{i, j}\left(\sum_{k=1}^{h_{j}} \beta^{2^{w_{j, k}}}\right)^{2^{i}}
$$




$$
\begin{aligned}
& =\sum_{i=0}^{m-1} a_{i} b_{i} \beta^{2^{i+1}}+\sum_{i=0}^{m-1} \sum_{j=1}^{v} x_{i, j}\left(\sum_{k=1}^{h_{j}} \beta^{2^{i+w_{j, k}}}\right) \\
& =\sum_{i=0}^{m-1} a_{i} b_{i} \beta^{2^{i+1}}+\sum_{j=1}^{v} \sum_{k=1}^{h_{j}}\left(\sum_{i=0}^{m-1} \beta^{2^{i+w_{j, k}}}\right) .
\end{aligned}
$$

Also, for even values of $m$, one has $v=\frac{m}{2}$ and $\delta_{v}=\delta_{v}^{2^{\frac{m}{2}}}$. This implies that in the normal basis representation of $\delta_{v}$, its $i$-th coordinate is equal to its $\left(\frac{m}{2}+i \bmod m\right)$-th coordinate. Thus, $h_{v}$ is even and one can write

$$
\delta_{v}=\sum_{k=1}^{\frac{h_{v}}{2}}\left(\beta^{2^{w_{v, k}}}+\beta^{2^{w_{v, k}+v}}\right), \quad v=\frac{m}{2} .
$$

Now, using (11) into Lemma 2 (for $m$ even) and using (10), we have the following theorem, where all indices and exponents are reduced modulo $m$.

Theorem 2. Let $A$ and $B$ be two elements of $G F\left(2^{m}\right)$ and $C$ be their product. Then

$$
C= \begin{cases}\sum_{i=0}^{m-1} a_{i} b_{i} \beta^{2^{i+1}}+\sum_{j=1}^{v} \sum_{k=1}^{h_{j}}\left(\sum_{i=0}^{m-1} x_{i, j} \beta^{2^{i+w_{j, k}}}\right), \quad \text { for m odd } \\ \sum_{i=0}^{m-1} a_{i} b_{i} \beta^{2^{i+1}}+\sum_{j=1}^{v-1} \sum_{k=1}^{h_{j}}\left(\sum_{i=0}^{m-1} x_{i, j} \beta^{2^{i+w_{j, k}}}\right)+F, \text { for m even }\end{cases}
$$

where

$$
F=\sum_{k=1}^{\frac{h_{v}}{2}} \sum_{i=0}^{v-1} x_{i, v}\left(\beta^{2^{i+w_{v, k}}}+\beta^{2^{i+w_{v, k}+v}}\right), \text { and } v=\frac{m}{2} .
$$

Note that for a normal basis, the representation of $\delta_{j}$ is fixed and so is $w_{j, k}, 1 \leq j \leq v, 1 \leq k \leq h_{j}$. Now, define

$$
\Delta w_{j, k} \triangleq w_{j, k}-w_{j, k-1}, 1 \leq j \leq v, 1 \leq k \leq h_{j}, w_{j, 0}=0
$$

where $w_{j, k}$ 's are as given in (9). For a particular normal basis, all $w_{j, k}$ 's are fixed. Hence, all $\Delta w_{j, k}$ 's need to be determined only at the time of choosing the basis. Using $\Delta w_{j, k}$ 's, below we present an efficient NB (ENB) multiplication algorithm over $G F\left(2^{m}\right)$ for odd values of $m$. The corresponding algorithm for even values of $m$ is shown in [18]. Also, an efficient scheme to compute $\Delta w_{j, k}$ 's is presented in 18 .

Algorithm 3 (ENB Multiplication for $m$ Odd)

Input: $A, B \in G F\left(2^{m}\right), \Delta w_{j, k} \in[0, m-1], 1 \leq j \leq v, 1 \leq k \leq h_{j}, v=\frac{m-1}{2}$

Output: $C=A B$

1. Initialize $C:=A \odot B, S_{A}:=A, S_{B}:=B$

2. $\quad C \gg 1$

3. $\quad$ For $j=1$ to $v\{$ 


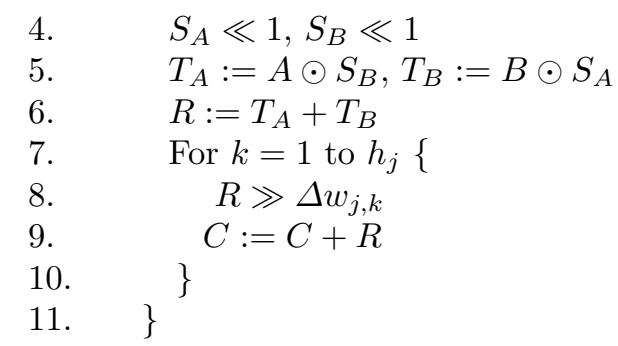

In the above algorithm, shifted values of $A$ and $B$ are stored in $S_{A}$ and $S_{B}$, respectively. In line $6, R \in G F\left(2^{m}\right)$ contains $\left(x_{0, j}, x_{1, j}, \cdots, x_{m-1, j}\right)$, i.e., $\sum_{i=0}^{m-1} x_{i, j} \beta^{2^{i}}$. Also, right cyclic shift of $R$ in lines 8 , corresponds to $\sum_{i=0}^{m-1} x_{i, j} \beta^{2^{i+w_{j, k}}}$. After the final iteration, $C$ is the normal basis representation of the required product $A B$. To illustrate the operation of the above algorithm, we present the following example.

Example 1. Consider the finite field $G F\left(2^{5}\right)$ generated by the irreducible polynomial $F(z)=z^{5}+z^{2}+1$ and let $\alpha$ be its root, i.e., $F(\alpha)=0$. We choose $\beta=\alpha^{5}$, then $\left\{\beta, \beta^{2}, \beta^{4}, \beta^{8}, \beta^{16}\right\}$ is a type 2 GNB. Here $m=5$, and $v=\frac{5-1}{2}=2$. Using Table 2 in 12 , one has

$$
\begin{aligned}
& \delta_{1}=\beta^{3}=\beta+\beta^{8}, \quad h_{1}=2,\left[w_{1, k}\right]_{k=1}^{h_{1}}=[0,3], \\
& \delta_{2}=\beta^{5}=\beta^{8}+\beta^{16}, h_{2}=2,\left[w_{2, k}\right]_{k=1}^{h_{2}}=[3,4] .
\end{aligned}
$$

Let $A=\beta^{2}+\beta^{4}+\beta^{8}=(01110)$ and $B=\beta+\beta^{4}+\beta^{16}=(10101)$ be two field elements. Table 1 shows contents of various variables of the algorithm as they are updated. The row with $j$ being '-' is for the initialization step (i.e., line 1) of the algorithm.

Table 1. Contents of variables in Algorithm 3 for multiplication of $A=(01110)$ and $B=(10101)$.

\begin{tabular}{|c|c|c|c|c|c|c|c|c|}
\hline$j$ & $S_{A}$ & $S_{B}$ & $T_{A}$ & $T_{B}$ & $k$ & $\Delta w_{j, k}$ & $R$ & $C$ \\
\hline- & 01110 & 10101 & - & - & - & - & - & 00010 \\
\hline \multirow{2}{*}{1} & 11100 & 01011 & 01010 & 10100 & & & 11110 & \\
& & & & & 1 & 0 & 11110 & 11100 \\
& & & & & 2 & 3 & 11011 & 00111 \\
\hline \multirow{2}{*}{2} & 11001 & 10110 & 00110 & 10001 & & & 10111 & \\
& & & & & 1 & 3 & 11110 & 11001 \\
& & & & & 2 & 1 & 01111 & 10110 \\
\hline
\end{tabular}

As it can be seen in Algorithm 3 all $\Delta w_{j, k}$ 's have to be pre-computed. In the above example, they are determined by calculating $\delta_{j}$ 's, which is essentially a multiplication process all by itself. For this multiplication, one can use either Algorithm 1 or Algorithm [2 However, an efficient scheme which does not need multiplication is presented in [18]. 


\subsection{Cost and Comparison}

In an effort to determine the cost of Algorithm 3 we give the dynamic instruction counts for its software implementation. We also consider the number of memory accesses to read the pre-computed values of $\Delta w_{j, k}$. For software implementation of the above algorithm, one would heavily rely on instructions, such as, XOR, AND and others which can be used to emulate cyclic shifts (in the C like programming language). XOR instructions are needed in lines 6 and 9 , which are repeated $v$ and $\sum_{j=1}^{v} h_{j}$ times, respectively. Since $v=\frac{m-1}{2}$ and $\sum_{j=1}^{v} h_{j}=\frac{C_{N}-1}{2}$ [10], the total number of XOR instructions is $\frac{1}{2}\left(C_{N}+m-1\right)\left\lceil\frac{m}{\omega}\right\rceil$. Because of the $\odot$ operations in lines 1 and 5 , one can also see that the above algorithm requires $m\left\lceil\frac{m}{\omega}\right\rceil$ AND instructions. We assume that each $i$-fold cyclic shift, $1 \leq i \leq m-1$, in lines 2,4 and 8 needs $\rho\left\lceil\frac{m}{\omega}\right\rceil$ instructions where $\rho$ is as defined earlier. In Algorithm 3 the number of cyclic shifts in lines 2, 4 and 8 are $1,2 v$ and $\sum_{j=1}^{v} h_{j}$, respectively. Thus, the total number of cyclic shifts in this algorithm is $1+2 v+\sum_{j=1}^{v} h_{j}=\frac{1}{2}\left(C_{N}+2 m-1\right)$ and so the total number of instructions to emulate cyclic shifts used in Algorithm 3 is $\frac{\rho}{2}\left(C_{N}+2 m-1\right)\left\lceil\frac{m}{\omega}\right\rceil$. Based on the above discussion, we have the following theorem.

Theorem 3. The dynamic instruction count for Algorithm 3 is given by

$$
\text { \#Instructions } \approx\left(\frac{1+\rho}{2} C_{N}+\frac{3+2 \rho}{2} m-\frac{2+\rho}{2}\right)\left\lceil\frac{m}{\omega}\right\rceil .
$$

For software implementation of Algorithm 3, if the loops are not unrolled and the values of $\Delta w_{j, k}$ 's are not hard-coded, one needs to store all these $\Delta w_{j, k}, 1 \leq$ $j \leq v, 1 \leq k \leq h_{j}$. Since the total number of $\Delta w_{j, k}$ 's is $\sum_{j=1}^{v} h_{j}$ and each $\Delta w_{j, k} \in[0, m-1]$ needs $\left\lceil\log _{2} m\right\rceil$ bits of memory, a total of about $\frac{C_{N}-1}{2}\left\lceil\log _{2} m\right\rceil$ bits of memory is needed to store the pre-computed $\Delta w_{j, k}$ 's.

Table 2. Comparison of multiplication algorithms in terms of number of instructions and memory requirements.

\begin{tabular}{|c|c|c|c|c|c|}
\hline \multirow{2}{*}{ Algorithms } & \multicolumn{3}{|c|}{ \# Instructions } & \multicolumn{2}{c|}{ Memory } \\
\cline { 2 - 6 } & XOR & AND & Others & Size in bits & \# Accesses \\
\hline Alg. 1 & $\frac{1}{4} m(t m-1)$ & $m(t m-1)$ & $2 \rho m\left\lceil\frac{m}{\omega}\right\rceil$ & $(t m-1)\left\lceil\log _{2} m\right\rceil$ & $2 m(t m-1)$ \\
\hline Alg. 2 & $(t m-1)\left\lceil\frac{m}{\omega}\right\rceil$ & $(t m-1)\left\lceil\frac{m}{\omega}\right\rceil$ & $2 \rho(t m-1)\left\lceil\frac{m}{\omega}\right\rceil$ & $\frac{t m}{2}\left\lceil\log _{2} m\right\rceil$ & $t m$ \\
\hline Alg. 3 & $\frac{1}{2}\left(C_{N}+m-2\right)\left\lceil\frac{m}{\omega}\right\rceil$ & $m\left\lceil\frac{m}{\omega}\right\rceil$ & $\frac{\rho}{2}\left(C_{N}+2 m-1\right)\left\lceil\frac{m}{\omega}\right\rceil$ & $\frac{C_{N}-1}{2}\left\lceil\log _{2} m\right\rceil$ & $\frac{C_{N}-1}{2}$ \\
\hline $\begin{array}{c}\text { Ratio of } \\
\text { Alg.2 2 to Alg. 3 }\end{array}$ & $\approx \frac{2 t}{t+1}$ & $\approx t$ & $\approx \frac{4 t}{t+1}$ & $\approx 1$ & $\approx 2$ \\
\hline
\end{tabular}

Table 2 compares the number of dynamic instructions of the three algorithms we have described so far. This table also gives memory sizes and numbers of memory accesses of these algorithms. As it can be seen in Table 2 both our proposed schemes (i.e., Algorithms 2] and [3) are superior to the conventional bit-level multiplication scheme (i.e., Algorithm 11). The final row of Table 2 gives approximate improvement factors of Algorithm 3 to Algorithm 2, A more detailed 
comparison of these two algorithms are given in Table 3 for the five binary fields recommended by NIST for ECDSA (elliptic curve digital signature algorithm) [13]. We have also coded these algorithms in software using the $\mathrm{C}$ programming language. Table 3 also shows timing (in $\mu s$ ) for these codes executed on Pentium III $533 \mathrm{MHz} \mathrm{PC}^{2}$. Our codes are parameterized in the sense that they can be used for various $m$ and $t$ without major modifications. For high speed implementation, the codes can be optimized for special values of $m$ and $t$.

Agnew et. al. in [1] have proposed a bit-serial architecture for the NB multiplication. Although their work has been targeted to hardware implementation, the main idea can be used for software implementation similar to the vector level method proposed here. For such a software implementation of 1, one would require $\left(C_{N}-1\right)\left\lceil\frac{m}{\omega}\right\rceil$ XOR instructions, $m\left\lceil\frac{m}{\omega}\right\rceil$ AND instructions, and $\rho\left(C_{N}+m-1\right)\left\lceil\frac{m}{\omega}\right\rceil$ other instructions. Thus, the dynamic instruction count would be $(\rho+1)\left(C_{N}+m-1\right)\left\lceil\frac{m}{\omega}\right\rceil$ which is about twice of that in Algorithm 3 (see Theorem [3). In [19], one can find software implementation of the NB multiplication for two special cases, namely, two optimal normal bases. The method used in [19] is similar to that of the NB multiplication of [1].

Some of the recently proposed polynomial basis multiplication algorithms, for example [6], [9], create a look-up table on the fly based on one of the inputs (say $B$ ) and yield significant speed-ups by processing a group of bits of the other input (i.e., $A$ ) at a time. At this point, it is not clear whether such a group-level processing of $A$ can be incorporated into our Algorithm 3 . However, if $m$ is a composite number, then one can essentially achieve similar kind of group-level processing by performing computations in the sub-fields. This idea is explored in the following section.

Table 3. Comparison of the proposed algorithms for binary fields recommended by NIST for ECDSA applications $(\omega=32)$.

\begin{tabular}{|c|c|c|c|c|c|c|c|c|c|c|}
\hline \multirow{3}{*}{\multicolumn{3}{|c|}{ Parameters }} & \multirow{2}{*}{\multicolumn{8}{|c|}{$\begin{array}{l}\text { Algorithm } 2 \\
\text { Algorithm } 3\end{array}$}} \\
\hline & & & & & & & & & & \\
\hline & & & \multicolumn{4}{|c|}{ \# Instructions } & \multicolumn{2}{|c|}{ Memory } & \multicolumn{2}{|c|}{ Timing } \\
\hline$m$ & $t$ & $C_{N}$ & XOR & AND & Others $/ \rho$ & Total $(\rho=4)$ & Size in bits & \# Accesses & in $\mu s$ & Ratio \\
\hline 163 & 4 & 645 & $\begin{array}{c}3906, \\
2418\end{array}$ & $\begin{array}{c}3906 \\
978\end{array}$ & $\begin{array}{l}7812 \\
2910\end{array}$ & $\begin{array}{c}39060 \\
15036\end{array}$ & $\begin{array}{c}2608, \\
2576\end{array}$ & $\begin{array}{l}652 \\
322\end{array}$ & $\begin{array}{c}307, \\
99\end{array}$ & $3.1: 1$ \\
\hline 233 & 2 & 465 & $\begin{array}{l}3720, \\
2784\end{array}$ & $\begin{array}{l}3720 \\
1864\end{array}$ & $\begin{array}{l}7440 \\
3720\end{array}$ & $\begin{array}{l}37200 \\
19528\end{array}$ & $\begin{array}{l}1864, \\
1856\end{array}$ & $\begin{array}{l}466, \\
232\end{array}$ & $\begin{array}{c}346, \\
126\end{array}$ & $2.75: 1$ \\
\hline 283 & 6 & 1677 & $\begin{array}{c}15273, \\
8811\end{array}$ & $\begin{array}{c}15273, \\
2547\end{array}$ & $\begin{array}{c}30546, \\
10089\end{array}$ & $\begin{array}{c}152730 \\
51714\end{array}$ & $\begin{array}{l}7641, \\
7542\end{array}$ & $\begin{array}{c}1698 \\
838\end{array}$ & $\begin{array}{c}1005 \\
318\end{array}$ & $3.16: 1$ \\
\hline 409 & 4 & 1629 & $\begin{array}{c}21255 \\
13234\end{array}$ & $\begin{array}{c}21255 \\
5317\end{array}$ & $\begin{array}{l}42510 \\
15899\end{array}$ & $\begin{array}{c}212550 \\
82147\end{array}$ & $\begin{array}{l}7362 \\
7326\end{array}$ & $\begin{array}{c}1636 \\
814\end{array}$ & $\begin{array}{c}1466, \\
473\end{array}$ & $3.1: 1$ \\
\hline 571 & 10 & 5637 & $\begin{array}{c}102762, \\
55854\end{array}$ & $\begin{array}{c}102762, \\
10278\end{array}$ & $\begin{array}{c}205524 \\
61002\end{array}$ & $\begin{array}{c}1027620 \\
310140\end{array}$ & $\begin{array}{l}28550, \\
28180\end{array}$ & $\begin{array}{l}5710 \\
2818\end{array}$ & $\begin{array}{l}8423, \\
2949\end{array}$ & 2.86:1 \\
\hline
\end{tabular}

\footnotetext{
${ }^{2}$ The PC has $64 \mathrm{M}$ bytes of RAM, $32 \mathrm{~K}$ bytes of L1 cache and $512 \mathrm{~K}$ bytes of L2 cache.
} 


\section{Efficient Composite Field NB Multiplication Algorithm}

In this section, we consider multiplications in the finite field $\operatorname{GF}\left(2^{m}\right)$ where $m$ is a composite number. These fields are referred to as composite fields and have been used in the recent past to develop efficient multiplication schemes [14, [15]. When these fields are to be used for elliptic curve crypto-systems, one must choose $m$ such that its factor are large enough to resist the attack described by Galbraith and Smart [4].

Lemma 3. [11] Let $\operatorname{gcd}\left(m_{1}, m_{2}\right)=1$. Let $N_{1}=\left\{\beta_{1}^{2^{j}} \mid 0 \leq j \leq m_{1}-1\right\}$ be a normal basis of $G F\left(2^{m_{1}}\right)$ over $G F(2)$. Then $N_{1}$ is also a normal basis of $G F\left(2^{m_{1} m_{2}}\right)$ over $G F\left(2^{m_{2}}\right)$.

Here, we consider composite fields with only two prime factors 3 (i.e., both $m_{1}$ and $m_{2}$ are prime). Thus, in the following we give all equations and algorithm for odd degrees (i.e., $m_{1}$ and $m_{2}$ ). The reader can easily extend it for even degrees using the results of the previous section. Also, the parameters, namely $\delta_{j}, h_{j}, v$, $\beta$, and $\Delta w_{j, k}$ of the previous section are used here in the context of the sub-fields $G F\left(2^{m_{1}}\right)$ and $G F\left(2^{m_{2}}\right)$ by putting an extra sub/superscript for example $\delta_{j}^{(1)}$ for $G F\left(2^{m_{1}}\right)$ and $\delta_{j}^{(2)}$ for $G F\left(2^{m_{2}}\right)$.

Let $A$ and $B$ be two elements of $G F\left(2^{m_{1}}\right)$ over $G F(2)$ and $C$ be their product. Then we have the following from [17.

$$
C=\sum_{i=0}^{m_{1}-1} a_{i} b_{i} \beta_{1}^{2^{i}}+\sum_{j=1}^{v_{1}} \sum_{k=1}^{h_{j}^{(1)}}\left(\sum_{i=0}^{m_{1}-1} y_{i, j} \beta_{1}^{2^{i+w_{j, k}^{(1)}}}\right), \text { for } m_{1} \text { odd }
$$

where

$$
\begin{gathered}
y_{i, j}=\left(a_{i}+a_{i+j}\right)\left(b_{i}+b_{i+j}\right), 1 \leq j \leq v_{1}, 0 \leq i \leq m_{1}-1, \\
v_{1}=\frac{m_{1}-1}{2}, \quad \beta_{1}^{2^{j}+1}=\sum_{k=1}^{h_{j}^{(1)}} \beta_{1}^{2^{w_{j, k}^{(1)}}} .
\end{gathered}
$$

By combining Lemma 3 with (14), the following is obtained.

Lemma 4. Let $A=\left(A_{0}, A_{1}, \cdots, A_{m_{1}-1}\right)$ and $B=\left(B_{0}, B_{1}, \cdots, B_{m_{1}-1}\right)$ be two elements of $G F\left(2^{m_{1} m_{2}}\right)$ over $G F\left(2^{m_{2}}\right)$ and $C$ be their product. Then

$$
C=\sum_{i=0}^{m_{1}-1} A_{i} B_{i} \beta_{1}^{2^{i}}+\sum_{j=1}^{v_{1}} \sum_{k=1}^{h_{j}^{(1)}}\left(\sum_{i=0}^{m_{1}-1} Y_{i, j} \beta_{1}^{2^{i+w_{j, k}^{(1)}}}\right), \text { for } m_{1} \text { odd }
$$

\footnotetext{
${ }^{3}$ This is important for elliptic curve crypto-systems. For such systems in today's security applications, the values of $m$ appear to be in the range of 160 to several hundreds only (571 as given in [13]). To avoid the attack of [4], one however may like to choose $m$ such that it has no small factors such as $2,3,5,7,11$. This basically makes one to choose $m$ as the product of two primes.
} 
where

$$
Y_{i, j}=\left(A_{i}+A_{i+j}\right)\left(B_{i}+B_{i+j}\right), 1 \leq j \leq v_{1}, 0 \leq i \leq m_{1}-1,
$$

and $A_{i}=\left(a_{i, 0}, a_{i, 1}, \cdots, a_{i, m_{2}-1}\right), B_{i}=\left(b_{i, 0}, b_{i, 1}, \cdots, b_{i, m_{2}-1}\right) \in G F\left(2^{m_{2}}\right)$ are sub-field coordinates of $A$ and $B$.

Lemma 4 leads to an algorithm for multiplication in composite fields using normal basis. The algorithm is stated below.

Algorithm 4 (ECFNB Multiplication of $G F\left(2^{m_{1} m_{2}}\right)$ over $G F\left(2^{m_{2}}\right)$ )

Input: $A, B \in G F\left(2^{m}\right), \Delta w_{j, k}^{(1)} \in\left[0, m_{1}-1\right], 1 \leq j \leq v_{1}, v_{1}=\frac{m_{1}-1}{2}, 1 \leq$ $k \leq h_{j}^{(1)}$

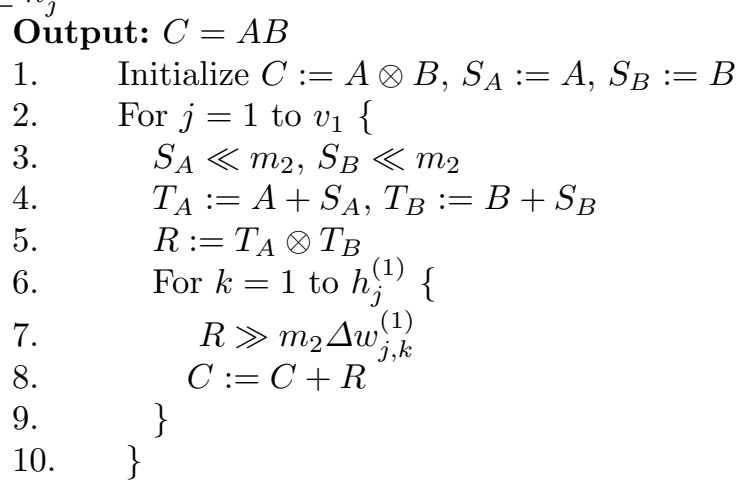

In lines 1 and 5 of Algorithm $4 . A \otimes B=\left(A_{0} B_{0}, A_{1} B_{1}, \cdots, A_{m_{1}-1} B_{m_{1}-1}\right)$ denotes parallel sub-field multiplications of $A$ and $B$. This sub-field multiplication can be implemented with an extension of Algorithm [3 such that it produces $m_{1}$ sub-field multiplications over $G F\left(2^{m_{2}}\right)$. This is shown in Algorithm [5 where $A \triangleright i$ (resp. $A \triangleleft i) 0 \leq i \leq m_{2}-1$, denotes an $i$-fold right (resp. left) sub-field cyclic shift of all sub-field elements of $A$, i.e., $A_{0}, A_{1}, \cdots, A_{m_{1}-1}$, respectively.

Algorithm 5 (Parallel Sub-Field Multiplication over $G F\left(2^{m_{2}}\right)$ )

Input: $A, B \in G F\left(2^{m}\right), \Delta w_{j, k}^{(2)} \in\left[0, m_{2}-1\right], 1 \leq j \leq v_{2}, 1 \leq k \leq h_{j}^{(2)}, v_{2}=$

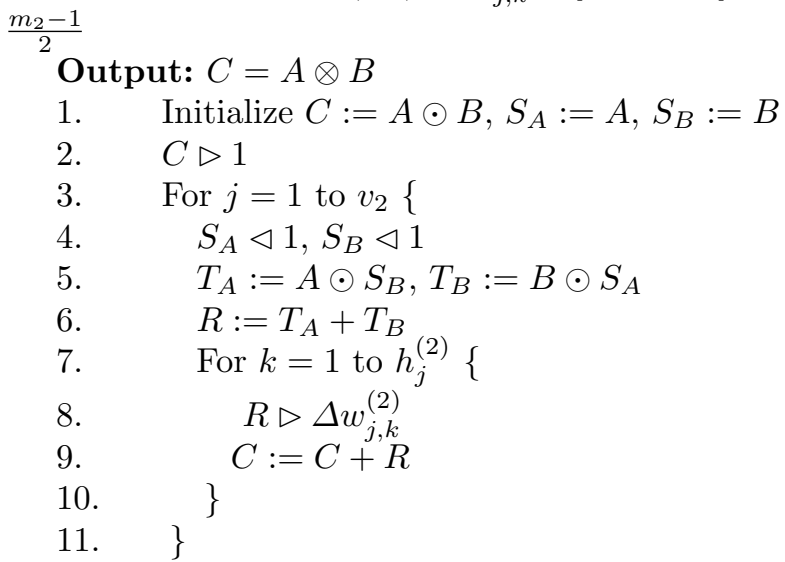


In order to obtain the cost of Algorithm 4 we need to evaluate the cost of Algorithm 5 which is called $1+v_{1}=\frac{m_{1}+1}{2}$ times by the former. Like Algorithm 3 one can determine the dynamic instruction counts of Algorithm 5 to be $\frac{1}{2}\left(C_{2}+\right.$ $\left.m_{2}-2\right)$ XOR, $m_{2}$ AND and $\frac{1}{2}\left(C_{2}+2 m_{2}-1\right)$ others to emulate cyclic shifts. The total cost of Algorithm 4 also depends on how sub-field elements, each of $m_{2}$ bits, are stored in registers. For the sake of simplicity we assume that an element of $G F\left(2^{m_{2}}\right)$ is stored in one $\omega$-bit register (for software implementation of elliptic curve crypto-systems with both $m_{1}$ and $m_{2}$ being prime, most general purpose processors would have $\omega$ bit registers where $\omega \geq m_{2}$ ). For $\omega=24$ and 32 , the best values of $m_{2}$ are those which have ONBs, i.e., 23 and 29, respectively. Thus, each element of $G F\left(2^{m}\right)$ needs $m_{1}$ registers and the cyclic shifts in lines 3 and 7 of Algorithm 4 are almost free of cost (or at best register renaming). Based on this assumption, we give the dynamic instruction counts of Algorithm 4 in Table 4 In this table, $\mu$ is the number of instructions needed for one sub-field cyclic shift in each register and it is 4 in the $\mathrm{C}$ programming language.

Table 4. Cost of Algorithm 4

\begin{tabular}{|c|c|c|}
\hline \multirow{3}{*}{ \# Instructions } & XOR & $\frac{m_{1}}{2}\left[\left(C_{1}+2 m_{1}-3\right)+\frac{\left(m_{1}+1\right)}{2}\left(C_{2}+m_{2}-2\right)\right]$ \\
\cline { 2 - 3 } & AND & $\frac{m_{1} m_{2}\left(m_{1}+1\right)}{2}$ \\
\cline { 2 - 3 } & Others & $\frac{\mu m_{1}}{4}\left(m_{1}+1\right)\left(C_{2}+2 m_{2}-1\right)$ \\
\hline \multirow{2}{*}{ Memory } & Size in bits & $\frac{C_{1}-1}{2}\left\lceil\log _{2} m_{1}\right\rceil+\frac{C_{2}-1}{2}\left\lceil\log _{2} m_{2}\right\rceil$ \\
\cline { 2 - 3 } & $\#$ Accesses & $\frac{C_{1}-1}{2}+\frac{\left(m_{1}+1\right)\left(C_{2}-1\right)}{4}$ \\
\hline
\end{tabular}

Table 5 shows the number of instructions and memory requirements of $\mathrm{Al}$ gorithm 4 for six different composite fields. These six fields are obtained by combining three $m_{1}$ 's and two $m_{2}$ 's. Algorithm 4 is also coded for these composite fields using the $\mathrm{C}$ programming language. The actual timing (in $\mu \mathrm{s}$ ) of Algorithm 4 executed on Pentium III $533 \mathrm{MHz}$ PC are also shown in Table 5 .

Table 5. Cost of Algorithm 4 for certain composite fields $(\mu=4)$.

\begin{tabular}{|c|c|c|c|c|c|c|c|c|c|c|c|}
\hline \multicolumn{9}{|c|}{ Parameters } & \multicolumn{4}{c|}{ \# Instructions } & \multicolumn{2}{c|}{ Memory } & Actual timing \\
\cline { 1 - 2 } & $m_{1}$ & $m_{2}$ & $C_{1}$ & $C_{2}$ & XOR & AND & Others & Total & Size in bits & \# Accesses & $($ in $\mu$ s $)$ \\
\hline 299 & 13 & 23 & 45 & 45 & 3445 & 2093 & 16380 & 21918 & 198 & 176 & 114 \\
\hline 377 & $"$ & 29 & $"$ & 57 & 4264 & 2639 & 20748 & 27651 & 228 & 218 & 150 \\
\hline 391 & 17 & 23 & 81 & 45 & 6001 & 3519 & 27540 & 37060 & 310 & 238 & 188 \\
\hline 437 & 19 & $"$ & 117 & $"$ & 7714 & 4370 & 34200 & 46284 & 400 & 278 & 249 \\
\hline 493 & 17 & 29 & 81 & 57 & 7378 & 4437 & 34884 & 46699 & 340 & 292 & 242 \\
\hline 551 & 19 & $"$ & 117 & $"$ & 9424 & 5510 & 43320 & 58254 & 430 & 338 & 309 \\
\hline
\end{tabular}




\section{Conclusions}

In this article, we have presented a number of software algorithms for normal basis multiplication over $G F\left(2^{m}\right)$. Both Algorithms 2 and 3 make maximal use of the full width of the data-path of the processor on which the software is to be executed and they provide significant speed-ups compared to the conventional bit-level multiplication scheme (i.e., Algorithm[1). Algorithms 2 and 3 are particularly suitable if $m$ is a prime. Such values of $m$ are of importance, especially for designing high speed crypto-systems based on Koblitz curves and for protecting elliptic curve crypto-systems against the attack of Galbraith and Smart [4]. Both Algorithms 2 and 3 have been coded for software implementation using $\mathrm{C}$, and our timing results show that Algorithm 3 is about $200 \%$ faster that Algorithm 2 These results are for those five Gaussian normal bases over the binary fields which NIST has described in their ECDSA document [13]. For the purpose of using NIST parameters, although we have presented our results for Gaussian normal bases, our algorithms are quite generic and can be used for any normal bases of $G F\left(2^{m}\right)$ over $G F(2)$.

We have also considered composite fields with $m=m_{1} \cdot m_{2}$. To avoid the attack of [4] on elliptic curve crypto-systems defined over these composite fields, we choose both $m_{1}$ and $m_{2}$ to be prime. We have presented an algorithm (i.e., Algorithm 4) for normal basis multiplication for $G F\left(2^{m}\right)$ over $G F\left(2^{m_{2}}\right)$. Our results show that for similar values of $m$, Algorithm 4 can be much more efficient than Algorithm 3. For example, the actual timing of Algorithm 3 is 318 microseconds for $G F\left(2^{283}\right)$ whereas the timing of Algorithm 4 is 114 micro-seconds for $G F\left(2^{299}\right)$. Composite fields also provide an added flexibility to hardwaresoftware co-design of finite field processors. For example, Algorithm 5 which is called by Algorithm 4 a total of $\frac{m_{1}+1}{2}$ times, can be implemented in hardware for small values of $m_{2}$, and Algorithm 4 can be embedded in a micro-controller which would give us a high speed, yet quite flexible, normal basis multiplier over very large fields.

\section{Acknowledgment}

The authors would like to thank Z. Zhang for his help with implementing the algorithms and getting their timing results.

\section{References}

1. G. B. Agnew, R. C. Mullin, I. M. Onyszchuk, and S. A. Vanstone. "An Implementation for a Fast Public-Key Cryptosystem". Journal of Cryptology, 3:63-79, 1991.

2. G. B. Agnew, R. C. Mullin, and S. A. Vanstone. "An Implementation of Elliptic Curve Cryptosystems Over $F_{2} 155$ ". IEEE J. Selected Areas in Communications, 11(5):804-813, June 1993.

3. D. W. Ash, I. F. Blake, and S. A. Vanstone. "Low Complexity Normal Bases". Discrete Applied Mathematics, 25:191-210, 1989. 
4. S. D. Galbraith and N. Smart. A Cryptographic Application of Weil Descent. In Proceedings of the Seventh IMA Conf. on Cryptography and Coding, LNCS 1764, pages 191-200. Springer-Verlag, 1999.

5. S. Gao and Jr. H. W. Lenstra. "Optimal Normal Bases". Designs, Codes and Cryptography, 2:315-323, 1992.

6. M. A. Hasan. Look-up Table-Based Large Finite Field Multiplication in Memory Constrained Cryptosystems. IEEE Transactions on Computers, 49:749-758, July 2000 .

7. M. A. Hasan, M. Z. Wang, and V. K. Bhargava. "A Modified Massey-Omura Parallel Multiplier for a Class of Finite Fields". IEEE Transactions on Computers, 42(10):1278-1280, Oct. 1993.

8. D. Johnson and A. Menezes. "The Elliptic Curve Digital Signature Algorithm (ECDSA)". Technical Report CORR 99-34, Dept. of C 83 O, University of Waterloo, Canada, August 23 1999. Updated: Feb. 24, 2000.

9. J. Lopez and R. Dahab. High Speed Software Multiplication in $F_{2^{m}}$. In Proceedings of Indocrypt 2000, pages 203-212. LNCS 1977, Springer, 2000.

10. Chung-Chin Lu. "A Search of Minimal Key Functions for Normal Basis Multipliers". IEEE Transactions on Computers, 46(5):588-592, May 1997.

11. A. J. Menezes, I. F. Blake, X. Gao, R. C. Mullin, S. A. Vanstone, and T. Yaghoobian. Applications of Finite Fields. Kluwer Academic Publishers, 1993.

12. R. C. Mullin, I. M. Onyszchuk, S. A. Vanstone, and R. M. Wilson. "Optimal Normal Bases in $G F\left(p^{n}\right)$ ". Discrete Applied Mathematics, 22:149-161, 1988/89.

13. National Institute of Standards and Technology. Digital Signature Standard. FIPS Publication 186-2, February 2000.

14. S. Oh, C. H. Kim, J. Lim, and D. H. Cheon. "Efficient Normal Basis Multipliers in Composite Fields". IEEE Transactions on Computers, 49(10):1133-1138, Oct. 2000.

15. C. Paar, P. Fleishmann, and P. Soria-Rodriguez. "Fast Arithmetic for Public-Key Algorithms in Galois Fields with Composite Exponents". IEEE Transactions on Computers, 48(10):1025-1034, Oct. 1999.

16. A. Reyhani-Masoleh and M. A. Hasan. "A Reduced Redundancy Massey-Omura Parallel Multiplier over $G F\left(2^{m}\right)$ ". In $20^{\text {th }}$ Biennial Symposium on Communications, pages 308-312, Kingston, Ontario, Canada, May 2000.

17. A. Reyhani-Masoleh and M. A. Hasan. "On Efficient Normal Basis Multiplication". In LNCS 1977 as Proceedings of Indocrypt 2000, pages 213-224, Calcutta, India, December 2000. Springer Verlag.

18. A. Reyhani-Masoleh and M. A. Hasan. "Fast Normal Basis Multiplication Using General Purpose Processors". Technical Report CORR 2001-25, Dept. of C $\& 3$ O, University of Waterloo, Canada, April 2001.

19. M. Rosing. Implementing Elliptic Curve Cryptography. Manning Publications Company, 1999.

20. B. Sunar and C. K. Koc. "An Efficient Optimal Normal Basis Type II Multiplier". IEEE Transactions on Computers, 50(1):83-88, Jan. 2001. 